\title{
Influência da Medicação Pré-Anestésica com Midazolam e Clonidina no Nível de Hipnose após Indução Anestésica com Propofol e Alfentanil em Crianças. Monitorização pelo Índice Bispectral *
}

\section{Influence of Midazolam and Clonidine Premedication on Hypnosis Level after Anesthetic Induction with Propofol and Alfentanil in Children. Bispectral Index Monitoring}

Eliana Marisa Ganem, TSA ${ }^{1}$, Norma Sueli Pinheiro Módolo, TSA ${ }^{2}$, Pedro Thadeu Galvão Vianna, TSA ${ }^{3}$, Yara Marcondes Machado Castiglia TSA ${ }^{3}$

\section{RESUMO}

Ganem EM, Módolo NSP, Vianna PTG, Castiglia YMM - Influência da Medicação Pré-Anestésica com Midazolam e Clonidina no Nível de Hipnose após Indução Anestésica com Propofol e Alfentanil em Crianças. Monitorização pelo Índice Bispectral

Justificativa e Objetivos - A medicação pré-anestésica (MPA) é adjuvante da anestesia e diminui tanto a necessidade de concentrações elevadas de anestésicos como a ansiedade perioperatória, produzindo amnésia e contribuindo para estabilidade hemodinâmica. Dentre as drogas administradas na MPA de crianças, encontram-se o midazolam e a clonidina. O objetivo desta pesquisa foi avaliar se a MPA com midazolam e clonidina exerce influência no nível de hipnose, avaliado pelo $B I S$, em crianças após indução anestésica com propofol e alfentanil.

Método - Participaram do estudo 30 pacientes, com idades entre 2 e 12 anos, estado físico ASA I, submetidos a cirurgias eletivas, que foram distribuídos em 3 grupos: G1 - sem MPA, G2 - midazolam $\left(0,5 \mathrm{mg} \cdot \mathrm{kg}^{-1}\right)$ e $\mathrm{G} 3$ - clonidina $\left(4 \mu \mathrm{g} \cdot \mathrm{kg}^{-1}\right)$, por via oral, 60 minutos antes da cirurgia. Todos os pacientes receberam alfentanil $\left(30 \mu \mathrm{g} \cdot \mathrm{kg}^{-1}\right)$, propofol $\left(3 \mathrm{mg} \cdot \mathrm{kg}^{-1}\right)$ e atracúrio $\left(0,5 \mathrm{mg} \cdot \mathrm{kg}^{-1}\right)$. Avaliou-se o valor derivado do BIS antes da indução da anestesia (M1) e após a intubação (M2). O método estatístico utilizado foi a análise de variância para idade, peso e altura, e análise de perfil para o BIS, sendo o valor de $p<0,05$ considerado significativo.

Resultados - Quando se comparou o mesmo momento (M1 ou M2) entre os três grupos, não foram observadas diferenças estatisticamente significativas. Quando se compararam os dois momentos de um mesmo grupo, M1 foi maior que M2 nos três grupos.

\footnotetext{
* Recebido do (Received from) CET/SBA do Departamento de Anestesiologia da Faculdade de Medicina de Botucatu (FMB - UNESP)

1. Professora Adjunta Livre-Docente do CET/SBA da FMB - UNESP

2. Professora Assistente Doutora do CET/SBA da FMB - UNESP

3. Professor (a) Titular do CET/SBA da FMB - UNSP
}

Apresentado (Submitted) em 16 de março de 2001

Aceito (Accepted) para publicação em 08 de agosto de 2001

Endereço para Correspondência (Mail to):

Dra. Eliana Marisa Ganem

Dept ${ }^{\circ}$ de Anestesiologia da FMB - UNESP

Distrito de Rubião Júnior

18618-970 Botucatu, SP

(c) Sociedade Brasileira de Anestesiologia, 2002
Conclusões - A medicação pré-anestésica com midazolam e clonidina não influenciou o nível de hipnose em crianças induzidas com propofol e alfentanil.

UNITERMOS - HIPNÓTICOS: alfentanil, propofol; MEDICAÇÃO PRÉ-ANESTÉSICA: clonidina, midazolam; MONITORIZAÇÃO: índice bispectral

\section{SUMMARY}

Ganem EM, Módolo NSP, Vianna PTG, Castiglia YMM - Influence of Midazolam and Clonidine Premedication on Hypnosis Level after Anesthetic Induction with Propofol and Alfentanil in Children. Bispectral Index Monitoring

Background and Objectives - Premedication is an anesthetic adjuvant which decreases both perioperative anxiety and the need for high anesthetic concentrations, in addition to producing amnesia and contributing to hemodynamic stability. Midazolam and clonidine are among preanesthetic drugs indicated for children. This study aimed at evaluating whether preanesthetic midazolam or clonidine influences hypnosis in children induced with propofol and alfentanil.

Methods - Participated in this study 30 patients aged 2 to 12 years, physical status ASA I, undergoing elective surgeries, who were divided into 3 groups: G1- without premedication, G2 - oral midazolam $\left(0.5 \mathrm{mg} \cdot \mathrm{kg}^{-1}\right)$, and G3 - oral clonidine (4 $\left.\mu \mathrm{g} . \mathrm{kg}^{-1}\right)$, both administered 60 minutes before anesthesia. All patients received alfentanil $\left(30 \mu \mathrm{g} \cdot \mathrm{kg}^{-1}\right)$, propofol $\left(3 \mathrm{mg} \cdot \mathrm{kg}^{-1}\right)$, and atracurium $\left(0.5 \mathrm{mg} \cdot \mathrm{kg}^{-1}\right)$. BIS values were evaluated before anesthesia induction (M1) and after tracheal intubation (M2). Analysis of variance was the statistical method used for age, weight, and height, and profile analysis was used for BIS values, considering significant $p<0.05$.

Results - In comparing the same moment (M1 or M2) among the three groups there were no significant differences. When comparing the two moments of a same group, M1 values were higher than $M 2$ values in all groups.

Conclusions - Midazolam and clonidine premedication have not influenced hypnosis level of propofol and alfentanil in children.

KEY WORDS - HYPNOTICS: alfentanil, propofol; MONITORING: bispectral index; PREANESTHETIC MEDICATION: clonidine, midazolam

\section{INTRODUÇÃO}

medicação pré-anestésica (MPA) é um coadjuvante da lanestesia que diminui tanto a necessidade de concentrações elevadas de anestésicos como a ansiedade perio- 
peratória, produzindo amnésia e contribuindo para estabilidade hemodinâmica no per-operatório ${ }^{1}$. Dentre as drogas administradas na MPA de crianças, encontram-se o midazolam, benzodiazepínico solúvel em água, sedativo eficaz em reduzir a ansiedade, e por isso amplamente utilizado $^{2}$, e a clonidina, agonista $\alpha_{2}$ adrenérgico que proporciona sedação pré-operatória ${ }^{3}$, diminuindo as necessidades de anestésicos durante a cirurgia ${ }^{4}$.

O índice bispectral (BIS), valor derivado do eletroencefalograma, tem-se mostrado medida quantificável dos efeitos hipnóticos das drogas anestésicas no sistema nervoso central $^{5,6}$. Representado por escala numérica que varia de 100 (acordado) a 0 (eletroencefalograma isoelétrico), quando utilizado durante a indução, a manutenção e a recuperação da anestesia de crianças ${ }^{7}$ apresenta valores idênticos aqueles dos adultos, tornando viável a sua utilização em pacientes pediátricos.

O objetivo desta pesquisa foi avaliar, pelo BIS, se a MPAcom midazolam e clonidina exerce influência no nível de hipnose de crianças após indução anestésica com propofol e alfentanil.

\section{MÉTODO}

Após a aprovação do Comitê de Ética em Pesquisa Clínica e o consentimento dos pais ou responsáveis, participaram do estudo 30 pacientes, de ambos os sexos, com idades entre 2 e 12 anos e estado físico ASAI, a serem submetidos a cirurgias eletivas. Os pacientes foram distribuídos por sorteio em 3 grupos (G1, G2 e G3) que se diferenciaram pela presença ou ausência de medicação pré-anestésica e pelo tipo de MPA.

Após jejum de 8 horas, os pacientes do G1 não receberam medicação pré-anestésica, os do G2 foram medicados com $0,5 \mathrm{mg} \cdot \mathrm{kg}^{-1}$ de midazolam e os do $\mathrm{G} 3$, com $4 \mu \mathrm{g} \mathrm{kg}^{-1}$ de clonidina (dose máxima de $150 \mu \mathrm{g} \cdot \mathrm{kg}^{-1}$ ), ambos por via oral, $60 \mathrm{mi}-$ nutos antes de serem encaminhados ao centro cirúrgico. $\mathrm{Na}$ sala de operação, após obtenção de acesso venoso, foi instalada a infusão de solução de Ringer com lactato, 4 $\mathrm{ml} \cdot \mathrm{kg}^{-1} \cdot \mathrm{h}^{-1}$, controlada por bomba de infusão contínua, e procedeu-se à monitorização com eletrocardioscópio em $D_{\|}$, aparelho de pressão arterial não invasiva, oxímetro de pulso, termômetro digital e aparelho que fornece valor derivado de Índice Bispectral. Iniciou-se a indução anestésica com alfentanil $\left(30 \mu \mathrm{g} \cdot \mathrm{kg}^{-1}\right)$ e propofol $\left(3 \mathrm{mg} \cdot \mathrm{kg}^{-1}\right)$. Para auxiliar a intubação, foram utilizados o atracúrio $\left(0,5 \mathrm{mg} \cdot \mathrm{kg}^{-1}\right)$ e a ventilação manual com oxigênio $\left(\mathrm{O}_{2}\right)$ a $100 \%$. Realizou-se, então, a intubação orotraqueal e a instalação de capnografia. Foram avaliados a freqüência cardíaca ( $F C$ ), a pressão arterial sistólica (PAS), a pressão arterial diastólica (PAD), a saturação de oxigênio pela hemoglobina $\left(\mathrm{SpO}_{2}\right)$ e o valor derivado do BIS antes da indução da anestesia (M1) e após a intubação, a qual se realizou 3 minutos após a indução (M2). Com a finalidade de comparar os grupos e realizar estudo estatístico de seus resultados, foram analisados a FC, a PAS, a PAD e o valor derivado do BIS, nos momentos M1 e M2.
O método estatístico utilizado foi a análise de variância para idade, peso e altura e análise de perfil para FC, PAS, PAD e BIS, sendo o valor de $p<0,05$ considerado significativo.

\section{RESULTADOS}

Os três grupos foram homogêneos com relação ao peso, à idade e à altura (Tabela I).

Tabela I - Dados Demográficos (Média \pm DP)

\begin{tabular}{lccc}
\hline & $\mathrm{G} 1$ & $\mathrm{G} 2$ & $\mathrm{G} 3$ \\
\hline $\mathrm{N}$ & 10 & 10 & 10 \\
Idade (anos) & $5,7 \pm 2,7$ & $5,8 \pm 3,5$ & $7,6 \pm 2,5$ \\
Peso $(\mathrm{kg})$ & $26 \pm 11$ & $22 \pm 10$ & $28 \pm 10$ \\
Altura $(\mathrm{cm})$ & $120 \pm 15$ & $114 \pm 22$ & $126 \pm 17$ \\
\hline
\end{tabular}

Não foram encontradas diferenças estatisticamente significativas nos valores de PAS, PAD, FC e BIS quando se comparou o mesmo momento (M1 ou M2) entre os três grupos de estudo (G1, G2 e G3) (Tabela II).

Tabela II - Pressão Arterial Sistólica (PAS), Pressão Arterial Diastólica (PAD), Freqüência Cardíaca (FC), Índice Bispectral (BIS) (Média \pm DP)

\begin{tabular}{|c|c|c|c|c|}
\hline & \multirow[t]{2}{*}{ Grupos } & \multicolumn{2}{|c|}{ Momentos } & \multirow[t]{2}{*}{ Estatística } \\
\hline & & M1 & M2 & \\
\hline & G1 & 108 & $91,5^{*}$ & $\mathrm{G} 1=\mathrm{G} 2=\mathrm{G} 3$ \\
\hline \multirow[t]{3}{*}{ PAS } & G2 & 100,5 & 96,5 & ${ }^{*} \mathrm{M} 1>\mathrm{M} 2$ \\
\hline & G3 & 108 & 96,5 & \\
\hline & G1 & 72,9 & $59^{*}$ & $\mathrm{G} 1=\mathrm{G} 2=\mathrm{G} 3$ \\
\hline \multirow[t]{3}{*}{ PAD } & G2 & 72,5 & 68 & ${ }^{*} \mathrm{M} 1>\mathrm{M} 2$ \\
\hline & G3 & 72,5 & $63,5^{*}$ & \\
\hline & G1 & 100 & 93 & $\mathrm{G} 1=\mathrm{G} 2=\mathrm{G} 3$ \\
\hline \multirow[t]{3}{*}{ FC } & G2 & 110 & 102 & \\
\hline & G3 & 94 & 91 & \\
\hline & G1 & 95 & $44^{*}$ & $\mathrm{G} 1=\mathrm{G} 2=\mathrm{G} 3$ \\
\hline \multirow[t]{2}{*}{ BIS } & G2 & 92 & $39^{*}$ & ${ }^{*} \mathrm{M} 1>\mathrm{M} 2$ \\
\hline & G3 & 92 & $37^{*}$ & \\
\hline
\end{tabular}

${ }^{*} p<0,05$

Ao estudar o comportamento dos momentos dentro de um mesmo grupo, constatou-se que a PAS e a PAD em G1 e G3 foram estatisticamente maiores em M1. AFC foi idêntica em ambos os momentos nos três grupos de estudo. E os valores do BIS foram menores em M2 nos três grupos (Tabela II).

\section{DISCUSSÃo}

Pelos nossos resultados, pudemos observar que a MPA com midazolam ou clonidina não exerceu influência no nível de hipnose, avaliada pelo BIS, em crianças, após a indução anestésica com propofol ( $\left.3 \mathrm{mg} \cdot \mathrm{kg}^{-1}\right)$ e alfentanil $\left(30 \mu \mathrm{g} \cdot \mathrm{kg}^{-1}\right)$.

Revista Brasileira de Anestesiologia Vol. 52, No 1, Janeiro - Fevereiro, 2002 
Escolheu-se o midazolam, como MPA, por ser a droga utilizada de rotina em nosso serviço, e a clonidina, pelos seus desejáveis efeitos sedativos ${ }^{3,8}$. A clonidina exerce sua ação por meio da ativação dos receptores $\alpha_{2}$-adrenérgicos do locus coeruleus, produzindo supressão de sua atividade e resultando em aumento da atividade de interneurônios inibitórios, como o da via do ácido gama aminobutírico (GABA), determinando depressão do sistema nervoso central (SNC) ${ }^{9}$. Em estudo prévio, em crianças, no qual foram utilizados o midazolam $0,5 \mathrm{mg} \cdot \mathrm{kg}^{-1}$ e a clonidina $4 \mu \mathrm{g} \cdot \mathrm{kg}^{-1}$, como MPA, observaram-se níveis idênticos de sedação e de redução da ansiedade, quando se utilizaram parâmetros clínicos para avaliação das duas medicações ${ }^{8}$.

Nós avaliamos a sedação dos pacientes por meio dos valores obtidos pelo BIS, e observamos que a presença de medicação e o tipo da mesma não influíram no nível de hipnose, visto que os valores do BIS foram iguais àqueles observados em crianças sem MPA.

Apesar de não existirem dados a respeito da biodisponibilidade da clonidina pela via oral em crianças ${ }^{9}$, estudos mostram que em 30 minutos após a administração de $4 \mu \mathrm{g} \cdot \mathrm{kg}^{-1} \mathrm{de}$ clonidina por essa via já se observa sedação que se intensifica com 60 minutos $^{3}$. E sessenta minutos foi o intervalo de tempo decorrido entre a administração da MPA e a chegada dos pacientes à sala de operação, portanto tempo suficiente para que a droga estivesse exercendo sua ação.

Escolheu-se o momento imediato à laringoscopia e à intubação traqueal para avaliar o nível de hipnose determinado pela dose de propofol e alfentanil administrada na indução porque estas manobras, que desencadeiam estímulos intensos, podem conduzir a aumento na pressão arterial, na freqüência cardíaca e nos níveis de catecolaminas circulantes em pacientes pediátricos ${ }^{10}$, quando a indução da anestesia é inadequada para bloquear tais estímulos. A clonidina, na dose de $4 \mu \mathrm{g} . \mathrm{kg}^{-1}$, evitou o aparecimento dos efeitos hemodinâmicos adversos ocasionados pela intubação traqueal, provavelmente porque reduziu os níveis de catecolaminas circulantes ${ }^{10}$. O alfentanil, em virtude de seu rápido início de ação, elevada margem de segurança e excelente estabilidade hemodinâmica, é muito utilizado na indução anestésica e em procedimentos de curta duração, em crianças. Em doses terapêuticas determina analgesia, sedação e ansiólise ${ }^{11}$. Em nossa pesquisa, utilizamos os valores de PAS, PAD e FC associados aos do BIS com a finalidade de se obterem parâmetros clínicos adicionais que assegurassem a qualidade da indução anestésica. O que pudemos observar foi redução na PAS e PAD após a indução da anestesia nos pacientes que não receberam MPA e naqueles que receberam clonidina. A ação da clonidina sobre o sistema cardiovascular pode ser periférica e central. A ativação dos receptores $\alpha_{2}$-adrenérgicos pré-sinapticos, nas terminações nervosas periféricas, inibindo a exocitose da noradrenalina, explica parcialmente o efeito hipotensor da droga ${ }^{12}$; entretanto, a ativação dos receptores pós-sinápticos, na musculatura dos vasos sangüíneos, produz vasoconstrição ${ }^{13}$. No SNC, a ativação dos receptores $\alpha_{2}$ diminui o efluxo simpático, com potenciali- zação da atividade nervosa parassimpática, induzindo hipotensão arterial $^{12}$.

O midazolam determina efeitos cardiocirculatórios mínimos, uma vez que os benzodiazepínicos exercem suas ações em sítios específicos dos $\mathrm{GABA}_{\mathrm{A}}$, e estes localizam-se exclusivamente nas terminações nervosas pós-sinápticas do SNC ${ }^{14}$.

Contudo, as crianças que não receberam MPA também apresentaram diminuição na PAS e PAD, mostrando que o propofol exerceu ação fundamental na redução da pressão arterial. O propofol determina hipotensão arterial porque produz relaxamento da musculatura lisa dos vasos por diminuição da resposta simpática vasoconstritora ${ }^{15}$.

Concluindo, nossos resultados sugerem que a MPA com midazolam na dose de $0,5 \mathrm{mg} \cdot \mathrm{kg}^{-1}$ e clonidina na dose de 4 $\mu \mathrm{g} \cdot \mathrm{kg}^{-1}$ não acentuou os efeitos hipnóticos do propofol na dose de $3 \mathrm{mg} \cdot \mathrm{kg}^{-1}$ associado ao alfentanil na dose de 30 $\mu \mathrm{g} . \mathrm{kg}^{-1}$ utilizados na indução da anestesia de pacientes pediátricos.

\section{Influence of Midazolam and Clonidine Premedication on Hypnosis Level after Anesthetic Induction with Propofol and Alfentanil in Children. Bispectral Index Monitoring}

Eliana Marisa Ganem, TSA, M.D., Norma Sueli Pinheiro Módolo, TSA, M.D., Pedro Thadeu Galvão Vianna, TSA, M.D., Yara Marcondes Machado Castiglia TSA, M.D.

\section{INTRODUCTION}

Premedication is an anesthetic adjuvant which decreases both perioperative anxiety and the need for high anesthetic concentrations, in addition to producing amnesia and contributing to hemodynamic stability ${ }^{1}$. Midazolam - a water-soluble benzodiazepine, effective in reducing anxiety and widely used $^{2}$ - and clonidine - an $\alpha_{2}$-adrenergic agonist providing preoperative sedation ${ }^{3}$ - are among preanesthetic drugs administered in children for decreasing perioperative anesthetic concentrations ${ }^{4}$.

EEG-derived bispectral index is a quantifiable measurement of the hypnotic effects of anesthetic drugs in the central nervous system ${ }^{5,6}$. Represented by a numeric scale from 100 (awaken) to 0 (isoelectric EEG) it shows, for anesthesia induction, maintenance and recovery in children, the same values found in adults, making it feasible for pediatric use. This study aimed at evaluating by BIS whether premedication with midazolam and cloinidine would influence hypnosis levels in children induced with propofol and alfentanil.

\section{METHODS}

After the Clinical Research Ethics Committee approval and the consent of parents or guardians, participated in this study 
30 patients of both genders, aged 2 to 12 years, physical status ASA I, submitted to elective surgeries. Patients were randomly distributed in 3 groups (G1, G2 and G3) according to the presence, absence or type of preanesthetic medication. After an 8-hour fast, G1 patients received no premedication, G2 patients were premedicated with $0.5 \mathrm{mg} . \mathrm{kg}^{-1}$ oral midazolam and $\mathrm{G} 3$ patients received $4 \mu \mathrm{g} . \mathrm{kg}^{-1}$ oral clonidine (maximum dose of $150 \mu \mathrm{g} . \mathrm{kg}^{-1}$ ), 60 minutes before being referred to the operating room. In the operating room, after installing a venous access, $4 \mathrm{ml} \cdot \mathrm{kg}^{-1} \cdot \mathrm{h}^{-1}$ lactated Ringer's in continuous infusion was started and monitoring consisted of ECG in $D_{\|}$, non-invasive blood pressure, pulse oximetry, digital thermometer and Bispectral Index. Anesthesia was induced with alfentanil $\left(30 \mu \mathrm{g} \cdot \mathrm{kg}^{-1}\right)$ and propofol $\left(3 \mathrm{mg} \cdot \mathrm{kg}^{-1}\right)$. Atracurium (0.5 $\left.\mathrm{mg} \cdot \mathrm{kg}^{-1}\right)$ and manual ventilation with $100 \%$ oxygen $\left(\mathrm{O}_{2}\right)$ were used to help tracheal intubation. Next, tracheal intubation was performed and capnography was installed. Heart rate (HR), systolic (SBP) and diastolic (DBP) blood pressure, oxygen saturation $\left(\mathrm{SpO}_{2}\right)$ and $\mathrm{BIS}$ were evaluated before anesthetic induction (M1) and after intubation, which was performed 3 minutes after induction (M2). Aiming at comparing groups and statistically studying results, HR, SBP, DBP and BIS were evaluated at $\mathrm{M} 1$ and $\mathrm{M} 2$.

Analysis of variance was adopted for age, weight and height, and profile analysis was used for HR, SBP, DBP and BIS, considering significant $p<0.05$.

\section{RESULTS}

The three groups were homogeneous in weight, age and height (Table I). There were no statistically significant differences in SBP, DBP, HR and BIS when comparing the same moment (M1 or M2) among groups (G1, G2 and G3) (Table II).

Table I - Demographics Data (Mean \pm SD)

\begin{tabular}{lccc}
\hline & $\mathrm{G} 1$ & $\mathrm{G} 2$ & $\mathrm{G} 3$ \\
\hline $\mathrm{N}$ & 10 & 10 & 10 \\
Age (years) & $5.7 \pm 2.7$ & $5.8 \pm 3.5$ & $7.6 \pm 2.5$ \\
Weight $(\mathrm{kg})$ & $26 \pm 11$ & $22 \pm 10$ & $28 \pm 10$ \\
Height $(\mathrm{cm})$ & $120 \pm 15$ & $114 \pm 22$ & $126 \pm 17$ \\
\hline
\end{tabular}

When comparing moments within a same group, SBP and DBP for $G 1$ and $G 3$ were statistically higher in M1. HR was identical in both moments for all groups. BIS values were lower in M2 for all groups (Table II).

\section{DISCUSSION}

In our study, premedication with midazolam or clonidine has not influenced BIS-evaluated hypnosis level in children after anesthetic induction with propofol $\left(3 \mathrm{mg} \cdot \mathrm{kg}^{-1}\right)$ and alfentanil $\left(30 \mu \mathrm{g} \cdot \mathrm{kg}^{-1}\right)$.

Midazolam was chosen because it is the routine drug in our hospital, and clonidine for its desirable sedative effects ${ }^{3,8}$.
Table II - Systolic Blood Pressure (SBP), Diastolic Blood Pressure (DBP), Heart Rate (HR), Bispectral Index (BIS) (Mean \pm SD)

\begin{tabular}{|c|c|c|c|c|}
\hline & Groups & Moments & & Statistics \\
\hline & & M1 & M2 & \\
\hline \multirow[t]{3}{*}{ SBP } & G1 & 108 & $91.5^{*}$ & $\mathrm{G} 1=\mathrm{G} 2=\mathrm{G} 3$ \\
\hline & G2 & 100.5 & 96.5 & * M1>M2 \\
\hline & G3 & 108 & 96.5 & \\
\hline \multirow[t]{3}{*}{ DBP } & G1 & 72.9 & $59^{*}$ & $\mathrm{G} 1=\mathrm{G} 2=\mathrm{G} 3$ \\
\hline & G2 & 72.5 & 68 & * M1>M2 \\
\hline & G3 & 72.5 & $63.5^{*}$ & \\
\hline \multirow[t]{3}{*}{$\mathrm{HR}$} & G1 & 100 & 93 & $\mathrm{G} 1=\mathrm{G} 2=\mathrm{G} 3$ \\
\hline & G2 & 110 & 102 & \\
\hline & G3 & 94 & 91 & \\
\hline \multirow[t]{3}{*}{ BIS } & G1 & 95 & $44^{*}$ & $\mathrm{G} 1=\mathrm{G} 2=\mathrm{G} 3$ \\
\hline & G2 & 92 & $39^{*}$ & ${ }^{*} \mathrm{M} 1>\mathrm{M} 2$ \\
\hline & G3 & 92 & $37^{*}$ & \\
\hline
\end{tabular}

${ }^{*} p<0.05$

Clonidine acts by activating locus coeruleus $\alpha_{2}$-adrenergic receptors, suppressing their activity and resulting in increased inhibitory interneurons, such as those found in the gamma aminubutiric acid (GABA) way, determining central nervous system (CNS) depression ${ }^{9}$. In a previous study in children premedicated with $0.5 \mathrm{mg} \cdot \mathrm{kg}^{-1}$ midazolam and $4 \mu \mathrm{g} \cdot \mathrm{kg}^{-1}$ clonidine identical levels of sedation and decreased anxiety were seen when clinical parameters were used to evaluate both medications ${ }^{8}$.

Our study has evaluated patients by BIS values and it was observed that the presence and type of medication have not influenced hypnosis levels since BIS values were similar to those observed in non-premedicated children.

Although the lack of data on oral clonidine bioavailability in children ${ }^{9}$, studies have shown that sedation is already present 30 minutes after $4 \mu \mathrm{g} . \mathrm{kg}^{-1}$ oral clonidine and is intensified at 60 minutes $^{3}$. And 60 minutes was the interval between premedication and referral to the operating room, that is, enough time for the drug to be fully active.

The moment immediately after laryngoscopy and tracheal intubation was chosen to evaluate hypnosis level determined by propofol and alfentanil induction dose because such maneuvers trigger major stimuli and may lead to an increase in blood pressure, heart rate and circulating catecholamines in pediatric patients ${ }^{10}$ when induction is inadequate to block such stimuli. Clonidine $\left(4 \mu \mathrm{g} \cdot \mathrm{kg}^{-1}\right)$ have prevented adverse hemodynamic effects caused by tracheal intubation, probably by decreasing circulating catecholamine levels ${ }^{10}$. Alfentanil, for its fast onset, high safety margin and excellent hemodynamic stability is widely used for anesthetic induction in short pediatric procedures. In therapeutic doses, it determines analgesia, sedation and ansiolysis ${ }^{11}$.

In our study, SBP, DBP and HR associated to BIS were used aiming at obtaining additional clinical parameters to assure anesthetic induction quality. It was observed that SBP and 
DBP decreased after induction in non-premedicated patients and in those receiving clonidine. Clonidine action on the cardiovascular system may be peripheral and central. Pre-synaptic $\alpha_{2}$-adrenergic receptors activation on peripheral nervous terminations inhibiting norepinephrine exocytosis partially explains drug's hypotensive effects ${ }^{12}$; however, the activation of pre-synaptic receptors in blood vessel muscles causes vasoconstriction ${ }^{13}$. In the CNS, the activation of $\alpha_{2}$-receptors decreases sympathetic eflux, increasing parasympathetic nervous activity and inducing hypotension ${ }^{12}$. Midazolam has minor cardiocirculatory effects since benzodiazepines act in specific $\mathrm{GABA}_{\mathrm{A}}$ sites which are exclusively located in CNS post-synaptic nervous terminations ${ }^{14}$. However, non-premedicated children have also shown decreased SBP and DBP, showing that propofol had a fundamental action in decreasing blood pressure. Propofol causes hypotension because it relaxes vessels' smooth muscles by decreasing vasoconstrictor sympathetic response ${ }^{15}$.

In conclusion, our results suggest that premedication with $0.5 \mathrm{mg} . \mathrm{kg}^{-1}$ midazolam and $4 \mu \mathrm{g} . \mathrm{kg}^{-1}$ clonidine have not increased the hypnotic effects of $3 \mathrm{mg} \cdot \mathrm{kg}^{-1}$ propofol associated to $30 \mu \mathrm{g} . \mathrm{kg}^{-1}$ alfentanil for anesthetic induction in pediatric patients.

\section{REFERÊNCIAS - REFERENCES}

01. Lichtor JL - The goals of premedication. Refresher Courses in Anesthesiology, 1995;23:141-156.

02. Alderson PJ, Lermam J - Oral premedication for paediatric ambulatory anaesthesia: a comparison of midazolam and ketamine. Can J Anaesth, 1994;41:221-226.

03. Mikawa K, Maekawa N, Nishina K et al - Efficacy of oral clonidine premedication in children. Anesthesiology, 1993;79:926-931.

04. Nishina K, Mikawa K, Maekawa N et al - The efficacy of clonidine for reducing perioperative haemodynamic changes and volatile anaesthetic requirements in children. Acta Anaesthesiol Scand, 1996; 40:746-751.

05. Liu J, Singh H, White PF - Electroencephalogram bispectral analysis predicts the depth of midazolam-induced sedation. Anesthesiology, 1996;84:64-69.

06. Liu J, Singh H, White PF - Electroencephalographic bispectral index correlates with intraoperative recall and depth of propofolinduced sedation. Anesth Analg, 1997;84:185-189.

07. Denman WT, Swanson EL, Rosow et al - Pediatric evaluation of bispectral index (BIS) monitor and correlation of BIS with end-tidal sevoflurane concentration in infants and children. Anesth Analg, 2000;90:872-877.

08. Lavrich PS, Hermann D, Pang LM et al - Clonidine as premedicant in children. Anesthesiology, 1996;85:A1085.

09. Nishina K, Mikawa K, Shiga M et al - Clonidine in paediatric anaesthesia. Paediatr Anaesth, 1999;9:187-202.

10. Mikawa K, Nishina K, Maekawa N et al - Attenuation of the catecholamine response to tracheal intubation with oral clonidine in children. Can J Anaesth, 1995;42;869-874.
11. Hiller A, Saaranivaara L - Injection pain, cardiovascular changes and recovery following induction of anaesthesia with propofol in combination with alfentanil or lignocaine in children. Acta Anaesthesiol Scand, 1992;36:564-568.

12. De Jonge A, Timmermans PB, Van Zweiten PA - Participation of cardiac presynaptic $\alpha_{2}$-adrenoceptors in the bradycardic effects of clonidine and analogues. Naunyn Schmiedebergs Arch Pharmacol, 1981;317:8-12.

13. Ruffolo JA - Distribution and function of peripheral adrenoceptores on the cardiovascular system. Pharmachol Biochem Behav, 1985;22:827-833.

14. Stoelting RK - Benzodiazepines, em: Stoelting RK - Pharmacology and Physiology in Anesthetic Practice, $3^{\text {rd }}$ Ed, Philadelphia: Lippincott-Raven Publishers, 1999;126-139.

15. Ronbinson BJ, Ebert TJ, O'Brien TJ et al - Mechanisms whereby propofol mediates peripheral vasodilatation in humans. Sympathoinhibition or direct vascular relaxation? Anesthesiology, 1997;86: 64-72.

\section{RESUMEN}

Ganem EM, Módolo NSP, Vianna PTG, Castiglia YMM - Influencia de la Medicación Pré-Anestésica con Midazolam y Clonidina en el Nivel de Hipnosis después de Inducción Anestésica con Propofol y Alfentanil en Niños. Monitorización por el Índice Bispectral

Justificativa y Objetivos - La medicación pré-anestésica (MPA) es adjuvante de la anestesia y diminuye tanto la necesidad de concentraciones elevadas de anestésicos, como la ansiedad perioperatoria, produciendo amnesia y contribuyendo para la estabilidad hemodinámica. De entre las drogas administradas en la MPA de niños, se encuentran el midazolam y la clonidina. El objetivo de esta pesquisa fue evaluar si la MPA con midazolam y clonidina ejerce influencia en el nivel de hipnosis, evaluado por el BIS, en niños después inducción anestésica con propofol e alfentanil.

Método - Participaron del estudio 30 pacientes, con edades entre 2 y 12 años, estado físico ASA I, sometidos a cirugías electivas, que fueron distribuidos en 3 grupos: G1 $\sin M P A, G 2$ midazolam $\left(0,5 \mathrm{mg} \cdot \mathrm{kg}^{-1}\right)$ y $\mathrm{G} 3$ - clonidina $\left(4 \mu \mathrm{g} \cdot \mathrm{kg}^{-1}\right)$, por vía oral, 60 minutos antes de la cirugía. Todos los pacientes recibieron alfentanil $\left(30 \mu \mathrm{g} \cdot \mathrm{kg}^{-1}\right)$, propofol $\left(3 \mathrm{mg} \cdot \mathrm{kg}^{-1}\right)$ y atracúrio $\left(0,5 \mathrm{mg} \cdot \mathrm{kg}^{-1}\right)$. Se evaluó el valor derivado del BIS antes de la inducción de la anestesia (M1) y después de la intubación (M2). El método estadístico utilizado fue la análisis de variancia para edad, peso y altura, y análisis de perfil para el $B I S$, siendo el valor de $p<0,05$ considerado significativo.

Resultados - Cuando se comparó el mismo momento (M1 o M2) entre los tres grupos, no fueron observadas diferencias estadísticamente significativas. Cuando se compararon los dos momentos de un mismo grupo, M1 fue mayor que M2 en los tres grupos.

Conclusiones - La MPA con midazolam y clonidina no influenció el nivel de hipnosis en niños inducidos con propofol y alfentanil. 